Educación Física y Ciencia, vol. 21, nº 2, e080, abril-junio 2019. ISSN 2314-2561

Universidad Nacional de La Plata.

Facultad de Humanidades y Ciencias de la Educación.

Departamento de Educación Física

\title{
Influencia de la pliometría basada en un Entrenamiento Intervalado de Alta Intensidad sobre la altura de salto y pico de potencia en futbolistas Sub - 17
}

\author{
The influence of High-Intensity Interval Training based plyometric exercise on jump height and peak power of Under-17 \\ male soccer players
}

Guillermo Daniel Barabona-Fuentes

Facultad de Educación, Universidad de Las Américas sede Viña del Mar, Chile

danielbarahonaf@gmail.com

Álvaro Huerta Ojeda

Facultad de Educación, Universidad de Las Américas sede Viña del Mar, Chile

achuertao@yahoo.es

Sergio Galdames Maliqueo

Facultad de Ciencias de la Actividad Física y del Deporte,

Universidad de Playa Ancha de Ciencias de la Educación, Chile

sergio.galdames@upla.cl

\section{Resumen:}

Introducción: Un entrenamiento pliométrico basado en HIIT que mejore la potencia muscular en futbolistas adolescentes, aún no ha sido del todo estudiado. Objetivo: Analizar el efecto de un entrenamiento pliométrico basado en HIIT sobre la potencia muscular en futbolistas Sub-17. Materiales y métodos: Veinticuatro futbolistas divididos en dos grupos fueron parte del estudio (Grupo Experimental [GE] n = 12 y Grupo Control $n=12$ ). Las variables fueron: altura (h) de salto y Pico de potencia (Pp) a través del Test de Sargent. El protocolo utilizado fue un entrenamiento pliométrico basado en HIIT. Para el análisis estadístico se utilizó la prueba t de Student y el tamaño del efecto fue calculado con la prueba d de Cohen. Resultados: La h en el Test de Sargent presentó un aumento significativo post intervención sólo para el GE $(\mathrm{p}<0.0001$; Tamaño del Efecto $=0.763)$. En relación a los Pp, sólo el GE presentó un aumento significativo post intervención $(\mathrm{p}<0.0001$; Tamaño del Efecto $=0.413)$. Conclusiones: Los resultados dejaron en evidencia que el entrenamiento pliométrico basado en HIIT es una herramienta efectiva para incrementar la h de salto y Pp en futbolistas adolescentes Sub-17.

Palabras Clave: Pliometría, Entrenamiento intervalado de alta intensidad, Adolescentes.

\section{Abstract:}

Introduction: A plyometric training based on HIIT that would improve the strength parameters in lower limbs of teenager soccer players has never been completely studied before. Objective: To analyze the effect of a HIIT based plyometric exercise program on muscle strength in Under-17 male soccer players. Materials and methods: Twenty-four Under-17 male soccer players were divided into two groups (Experimental Group [GE] n=12 and Control Group n=12.) The variables used were: The jump (h) height and the Peak power $(\mathrm{Pp})$ through the Sargent Jump Test. The protocol used was a HIIT based plyometric exercise program. The Student's T Test and the Effect Size were used for statistical analysis and the results were calculated with a Cohen's D. Results: Post intervention, only the GE ( $<<0.0001$; Effect Size $=0.763)$. showed a significant increase in the h of the Sargent Jump Test. In terms of the Pp, only the GE presented a significant increase post intervention $(\mathrm{p}<0.0001$; Effect Size $=0.413)$. Conclusions: The results revealed that a HIIT based plyometric exercise program is an effective tool to improve the height and the $\mathrm{Pp}$ in $\mathrm{h}$ of Under-17 male soccer players.

KEYWORDS: Plyometric training, High-Intensity Interval Training, Adolescents. 


\section{INTRODUCCIÓN}

En la actualidad, el entrenamiento deportivo tiene su base en el desarrollo de la capacidad de fuerza (Bautista, Chirosa, Chirosa, Martín, González, \& Robertson 2014; Huerta, Chirosa, Guisado, Chirosa, \& Cáceres, 2016a; Huerta, Chirosa, Guisado, Chirosa, \& Cáceres, 2016b). En relación a ello, se ha evidenciado que un mayor desarrollo de fuerza producto de los distintos sistemas de entrenamiento, junto con mejorar la salud metabólica de los sujetos, también aumentan el rendimiento deportivo, el cual se ha visto manifestado en una mayor altura de salto (Ingle, Stephenson, \& Sandercock, 2016). Por lo tanto, y de manera más específica, desarrollar altos niveles de fuerza y potencia muscular en futbolistas juveniles se ha convertido en un proceso fundamental para enfrentar distintas situaciones de juego (Chelly, Hermassi, Aouadi, \& Shephard, 2014; Comfort, Stewart, Bloom, \& Clarkson, 2014; Ozbar, Ates, \& Agopyan, 2014). Por el contrario, una disminución de fuerza muscular, movilidad articular y control neuromuscular, cambian el comportamiento funcional de un sujeto, limitando el rendimiento y desencadenando posiblemente una lesión (Ceroni, Martin, Delhumeau, \& Farpour-Lambert, 2012).

Por lo anterior, un entrenamiento eficiente de la potencia muscular y la transferencia de ésta para las tareas específicas del deporte, es un objetivo clave en la preparación de los atletas que participan en deportes de equipo (Teo, Newton, Newton, Dempsey, \& Fairchild, 2016). Se ha evidenciado que una amplia producción de potencia muscular y un alto nivel de rendimiento anaeróbico son componentes críticos para los futbolistas (Chelly et al., 2014). Cualquiera sea el método que se utilice para el progreso de la potencia muscular (sentadillas, levantamiento de pesas estilo olímpico, velocidad resistida, pliometría, etc), favorece el desarrollo de la potencia, desencadenando mejorías en el rendimiento deportivo, principalmente en las tareas propias de los deporte de equipo (Teo et al., 2016). En el caso de los futbolistas, un aumento en la potencia muscular se asocia a incrementos en la capacidad de salto y velocidad (de Hoyo et al., 2016; Teo et al., 2016).

En relación con los métodos de entrenamiento, la pliometría es uno de los sistemas más utilizados para mejorar la fuerza muscular (Chelly et al., 2014; Roopchand-Martin, \& Lue-Chin, 2010). Esta metodología hace referencia a una amplia gama de ejercicios que emplean el entrenamiento en base a saltos (de Hoyo et al., 2016). En este sentido, han sido numerosos los estudios que han utilizado el entrenamiento pliométrico para el desarrollo de la potencia muscular (Chelly et al., 2010; Lloyd, Radnor, De Ste Croix, Cronin, \& Oliver, 2016; Mackala, \& Fostiak, 2015; McKay, \& Henschke, 2012; Michailidis et al. 2013; RoopchandMartin, \& Lue-Chin, 2010; Sohnlein, Muller, \& Stoggl, 2014). Lo anterior, se debe a que el método pliométrico ha sido identificado como un componente altamente transferible hacia incrementos en potencia muscular y adaptaciones neuromusculares específicas (Loturco et al., 2015). Puntualmente, en futbolistas, se ha evidenciado un aumento en las habilidades motoras de aceleración, velocidad y salto, independientemente de la edad en que se encuentren (Lloyd et al., 2016; Loturco et al., 2015; McKay, \& Henschke, 2012; Michailidis et al., 2013).

Junto al método pliométrico, los entrenadores y preparadores físicos de futbolistas, también han utilizado otros sistemas de entrenamiento para mejorar la condición física (de Hoyo et al., 2016; Franco-Marquez et al., 2015; Lloyd et al., 2016). Un método ampliamente utilizado es el entrenamiento intervalado a alta intensidad (HIIT) (Brocherie, Girard, Faiss, \& Millet, 2015; Sperlich et al., 2011). Este método ha sido ampliamente utilizado, por tal razón, se han llegado a realizar submanifestaciones; entre ellas se encuentran HIIT con beneficios metabólicos y HIIT con beneficios neuromuscular (Abe et al., 2015; Kohn, Essen-Gustavsson, \& Myburgh, 2011). Es importante mencionar, que en la mayoría de las investigaciones consultadas el HIIT fue utilizado con la finalidad de mejorar el consumo máximo de oxígeno (VO2max) (Costigan, Eather, Plotnikoff, Taaffe, \& Lubans, 2015; Huerta, Galdames, Cataldo, Barahona, Rozas, \& Cáceres, 2017; Logan et al., 2016; Sperlich et al., 2011). En un estudio desarrollado por Logan et al. (2016), se muestra como el HIIT generó mejorías en la aptitud física de adolescentes, específicamente el VO2max. De forma paralela, en algunas investigaciones se ha evidenciado que el HIIT es una metodología apropiada para mejorar la 
potencia de los miembros inferiores (Brocherie et al., 2015; Naimo et al., 2015). Ejemplo de lo anterior, en una investigación presentada por Naimo et al. (2015) se reportó que los jugadores de hockey sobre hielo obtienen efectos positivos en el espesor del músculo, un incremento en la potencia y rendimiento posterior a la utilización de un HITT. También, se ha recomendado que el HIIT incorpore ejercicios explosivos y de fuerza para mejorar la aptitud muscular, la potencia y los factores relacionados con resistencia a la fatiga (Brocherie et al., 2015; Costigan et al., 2015). Por lo anteriormente expuesto, se puede observar que tanto el método pliométrico como el HIIT mejoran los componentes de la condición física (Lloyd et al., 2016; Loturco et al., 2015; McKay, \& Henschke, 2012; Michailidis et al., 2013; Brocherie et al., 2015; Costigan et al., 2015). Sin embargo, hasta donde sabemos, la incorporación de un entrenamiento pliométrico basado en HIIT que mejoren los parámetros neuromusculares, específicamente la potencia de los miembros inferiores en futbolistas adolescentes es escasa, por lo que no se tiene conocimiento respecto a un protocolo de ejercicios óptimos de ésta índole, que provoquen adaptaciones fisiológicas en deportistas adolescentes.

Consecuencia de lo anterior, el objetivo principal del estudio fue conocer el efecto de un programa de entrenamiento pliométrico basado en un HIIT sobre la potencia muscular en futbolistas Sub- 17.

\section{MATERIAL Y MÉtodo}

\section{Aproximación experimental al estudio}

En este estudio, la muestra fue de 24 futbolistas aficionados Sub-17 divididos en dos grupos. El primero de ellos, definido como Grupo Experimental (GE) estuvo constituido por 12 sujetos ( $\mathrm{n}=12$ ), mientras que el segundo, fue definido como Grupo Control (GC) y fue constituido por 12 sujetos $(\mathrm{n}=12)$. El criterio de inclusión fue que todos los sujetos incluidos en el estudio, debían pertenecer a la categoría de ftbol Sub- 17. Para la aplicación del protocolo, se utilizó un diseo cuasi experimental con pre test y post test, mientras que el protocolo aplicado fue un entrenamiento pliométrico basado en HIIT. Entre el pre test y el post test hubo ocho semanas de separación. Antes de comenzar el estudio, a todos los sujetos se les midió el peso ( $\mathrm{Kg})$ y la estatura $(\mathrm{cm})$. A cada uno de los participantes de la investigación se le solicitó que se abstuvieran de ingerir cafeína, medicamentos y cualquier sustancia que incrementara el metabolismo 48 horas antes del pre test y post test.

\section{SuJETOS}

Veinticuatro futbolistas Sub-17 de la provincia de Quillota, Chile (edad: 14,3 \pm 0,7 años; peso: 62,7 \pm 13,0 Kg; estatura: $165,9 \pm 0,0 \mathrm{~cm}$, índice de masa corporal (IMC): $22,5 \pm 3,1 \mathrm{Kg} / \mathrm{m} 2$ ) (Tabla 1) fueron parte del estudio. Los veinticuatro futbolistas Sub-17 fueron designados en GE y GC al azar por los investigadores. Tanto los sujetos como los tutores fueron informados del objetivo de estudio y de los posibles riesgos del experimento, todos firmaron un consentimiento y asentimiento informado antes de la aplicación del protocolo. El consentimiento informado, el asentimiento informado y el estudio fueron aprobados por el Comité de Investigación Humana de la Universidad de Playa Ancha de Ciencias de la Educación, Chile. Tanto el consentimiento informado como el asentimiento informado, fueron firmados por los tutores y sujetos intervenidos respectivamente. 
TABLA 1

Características de la muestra (medias $\pm \mathrm{DS})$

\begin{tabular}{lcc}
\hline & Grupo Experimental $(\mathrm{n}=12)$ & Grupo Control $(\mathrm{n}=12)$ \\
\hline Edad (años) & $14 \pm 0,7$ & $15,3 \pm 1,2$ \\
Estatura (cm) & $164,4 \pm 0,1$ & $166,6 \pm 0,7$ \\
Peso (Kg) & $61,0 \pm 13,5$ & $64,3 \pm 9,7$ \\
IMC & $22,1 \pm 3,1$ & $23,0 \pm 3,1$ \\
\hline IMC (índice de masa corporal); DS (desviación estándar).
\end{tabular}

\section{INSTRUMENTOS}

Para la caracterización de la muestra, el peso y la estatura se midieron con balanza Seca $807^{\circ}$ y Estadiómetro Seca $213^{\circ}$. Mientras que para calcular la altura de salto y el nivel de potencia muscular se utilizó el Test de Sargent (de Salles, Vasconcellos, de Salles, Fonseca, \& Dantas, 2012) y la fórmula matemática de Sayers (Lara-Sanchez, Zagalaz, Berdejo-Del-Fresno, \& Martinez-Lopez, 2011) respectivamente.

Calentamiento estandarizado. Para la evaluación del pre test y post test, además de las ocho semanas de aplicación del entrenamiento pliométrico basado en HIIT, el calentamiento estandarizado consistió en 5 minutos de trote, más cinco minutos de movimientos articulares entre ellos (circunducción de hombros, tronco, cadera, tobillos) perteneciente a los miembros superiores e inferiores respectivamente.

\section{Pre test Y POST TEST}

Para evaluar la altura de salto y potencia muscular antes y después de aplicado el entrenamiento pliométrico basado en HIIT, se aplicó el Test de Sargent. En esta evaluación los ejecutantes tuvieron tres intentos de salto, dejando el mejor registro obtenido. Una vez obtenida la altura de salto a través del Test de Sargent, y masa $(\mathrm{Kg})$, se recurrió a utilizar la fórmula propuesta por Sayers (Lara-Sanchez et al. 2011).

Potencia $(\mathrm{W})=\left(51,9^{*} \mathrm{~h}(\mathrm{~cm})\right)+\left(48,9^{*}\right.$ masa $\left.(\mathrm{Kg})\right)-2007$.

W (Watts); h (altura de salto); cm (centímetros); Kg (Kilogramos).

\section{Tratamiento}

Antes de comenzar con las sesiones del estudio, se llevó a cabo una intervención piloto. Dicha intervención tuvo por finalidad que los sujetos del GE tuvieran familiarización con las variables y lenguaje específico del entrenamiento, es decir, el reconocimiento de términos como series, repeticiones, tiempo, escala de esfuerzo percibido, entre otras.

El entrenamiento pliométrico basado en HIIT aplicado al GE, tuvo por objetivo incrementar la potencia muscular. El programa de entrenamiento tuvo una duración de 16 sesiones, mientras que la frecuencia fue de dos sesiones de entrenamiento por semana, y cada sesión tuvo una duración entre 35 y 41 minutos aproximadamente.

\section{MÉtodo de ENTRENAMIENTo PLIOMÉtrico EN BASE A HIIT}

Una vez realizada la división de la muestra, los participantes comenzaron el entrenamiento, el GE realizaba el tratamiento consistente en un entrenamiento pliométrico basado en HIIT de manera complementaria a su entrenamiento habitual. Mientras que el GC siguió su entrenamiento rutinario de fútbol enfocado principalmente en los aspectos técnicos y tácticos propios de la disciplina.

El entrenamiento consistió en una variedad de ejercicios de saltos que tuvieron una duración de ocho semanas con una frecuencia de dos sesiones por semana. Esta variedad de saltos iba de dos a cuatro ejercicios 
de saltos dependiendo de la sesión. Fue así como en la sesión 1,2, 3 y 4 se ejecutaron saltos de cuerda, skipping, jumping jacks y sentadillas con salto. Cada una de estas sesiones tuvo un total de 3 series por sesión, y cada serie incorporó 15 segundos (s) de saltar la cuerda, $15 \mathrm{~s}$ de skipping, $15 \mathrm{~s}$ de jumping jacks y $15 \mathrm{~s}$ de sentadillas con salto; la pausa entre ejercicio y entre serie fue de $60 \mathrm{~s}$. En las sesiones 5, 6, 7 y 8 se ejecutaron saltos de valla-plataforma $(65 \mathrm{~cm}$ de h) y salto a plataforma $(65 \mathrm{~cm}$ de h). Cada una de estas sesiones tuvo un total de 6 series por sesión, y cada serie incorporó $30 \mathrm{~s}$ de saltos de valla-plataforma $(65 \mathrm{~cm} \mathrm{de} \mathrm{h})$ y $30 \mathrm{~s}$ de salto a plataforma $(65 \mathrm{~cm}$ de h); la pausa entre ejercicio y entre serie fue de $60 \mathrm{~s}$. En las sesiones 9, 10, 11 y 12 se ejecutaron saltos laterales y salto en escalera de coordinación-plataforma $(65 \mathrm{~cm} \mathrm{de} \mathrm{h})$. Cada una de estas sesiones tuvo un total de 10 series por sesión, y cada serie incorporó $30 \mathrm{~s}$ de saltos laterales y $30 \mathrm{~s}$ de salto en escaleras de coordinación-plataforma $(65 \mathrm{~cm} \mathrm{de} \mathrm{h})$; la pausa entre ejercicio y entre serie fue de $30 \mathrm{~s}$. Finalmente, en las sesiones 13, 14, 15 y 16 se ejecutaron sentadillas con salto, saltos de valla-plataforma (65 $\mathrm{cm}$ de h), salto a plataforma ( $65 \mathrm{~cm}$ de h) y saltos laterales. Cada una de estas sesiones tuvo un total de 7 series por sesión, y cada serie incorporó $30 \mathrm{~s}$ de sentadilla con salto, $30 \mathrm{~s}$ de saltos valla-plataforma $(65 \mathrm{~cm} \mathrm{de} \mathrm{h})$, $30 \mathrm{~s}$ de salto a plataforma $(65 \mathrm{~cm}$ de h) y $30 \mathrm{~s}$ saltos laterales; la pausa entre ejercicio y entre serie fue de 15 s. Cabe destacar que la variedad de saltos, tuvo por finalidad evitar la monotonía del entrenamiento como también buscar la progresión en la intensidad, es así como los ejercicios de saltos fueron de lo más simple a lo más complejo (Davies, Riemann, \& Manske, 2015; Ebben, Fauth, Garceau, \& Petushek, 2011). Mientras que un aumento de volumen de las cargas por cada cuatro sesiones tuvo por finalidad conseguir un proceso de adaptación en cada sujeto como también disminuir el riesgo de lesiones o sobreentrenamiento (Davies et al. 2015), siguiendo de esta forma el principio de la progresión de las cargas (Lorenz, \& Morrison, 2015). (Ver Tabla 2).

TABLA 2

Programa de entrenamiento pliométrico basado en HIIT.

\begin{tabular}{|c|c|c|c|c|c|c|c|c|c|}
\hline \multicolumn{10}{|c|}{ Ejercicios usa dos en el entrenamiento pliométrice basados en HIIT } \\
\hline & & Saltarla cuerda & Skipping & Jumping jacks & Ss & Salto $\mathrm{Vp}$ & Salto $\mathrm{P}$ & Saltos laterales & Saltos en Ec - P \\
\hline & & $\begin{array}{c}\text { relación } \\
\text { trabajo-pausa }\end{array}$ & $\begin{array}{c}\text { relación } \\
\text { trabajo-pausa }\end{array}$ & $\begin{array}{c}\text { relación } \\
\text { trabajo-pausa }\end{array}$ & $\begin{array}{c}\text { relación } \\
\text { trabajo-pausa }\end{array}$ & $\begin{array}{c}\text { relación } \\
\text { trabajo-pausa }\end{array}$ & $\begin{array}{c}\text { relación } \\
\text { trabajo-pausa }\end{array}$ & $\begin{array}{c}\text { relación } \\
\text { trabajo-pausa }\end{array}$ & $\begin{array}{c}\text { relación } \\
\text { trabajo-pausa }\end{array}$ \\
\hline \multicolumn{10}{|c|}{ Sentido de la serie } \\
\hline Sesión & Series & & & & & & & & \\
\hline 1 & 3 & $15 s-60 s$ & $15 s-60 s$ & $15 s-60 s$ & $15 s-60 s$ & & & & \\
\hline 2 & 3 & $15 s-60 s$ & $15 s-60 s$ & $15 s-60 s$ & $15 s-60 s$ & & & & \\
\hline 3 & 3 & $15 s-60 s$ & $15 s-60 s$ & $15 s-60 s$ & $15 s-60 s$ & & & & \\
\hline 4 & 3 & $15 s-60 s$ & $15 s-60 s$ & $15 s-60 s$ & $15 s-60 s$ & & & & \\
\hline \multicolumn{10}{|c|}{ Sentido de la serie } \\
\hline Sesión & Series & & & & & & & & \\
\hline 5 & 6 & & & & & $30 s-60 s$ & $30 s-60 s$ & & \\
\hline 6 & 6 & & & & & $30 s-60 s$ & $30 s-60 s$ & & \\
\hline 7 & 6 & & & & & $30 s-60 s$ & $30 s-60 s$ & & \\
\hline 8 & 6 & & & & & $30 s-60 s$ & $30 s-60 s$ & & \\
\hline \multicolumn{10}{|c|}{ Sentido de la serie } \\
\hline Sesión & Series & & & & & & & & \\
\hline 9 & 10 & & & & & & & $30 s-30 s$ & $30 s-30 s$ \\
\hline 10 & 10 & & & & & & & $30 s-30 s$ & $30 s-30 s$ \\
\hline 11 & 10 & & & & & & & $30 s-30 s$ & $30 s-30 s$ \\
\hline 12 & 10 & & & & & & & $30 s-30 s$ & $30 s-30 s$ \\
\hline \multicolumn{10}{|c|}{ Sentido de la serie } \\
\hline Sesión & Series & & & & & & & & \\
\hline 13 & 7 & & & & $30 s-15 s$ & $30 s-15 s$ & $30 \mathrm{~s}-15 \mathrm{~s}$ & $30 s-15 s$ & \\
\hline 14 & 7 & & & & $30 s-15 s$ & $30 \mathrm{~s}-15 \mathrm{~s}$ & $30 s-15 s$ & $30 s-15 s$ & \\
\hline 15 & 7 & & & & $30 s-15 s$ & $30 s-15 s$ & $30 s-15 s$ & $30 s-15 s$ & \\
\hline 16 & 7 & 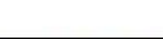 & 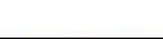 & 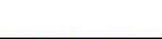 & $30 s-15 s$ & $30 s-15 s$ & $30 s-15 s$ & $30 s-15 s$ & \\
\hline
\end{tabular}

Cabe mencionar que cada uno de los ejercicios se realizó con una escala de esfuerzo percibido (EEP) (Borg, 1998). Este EEP va en una escala de 0 a 10, siendo una EEP 0 (reposo), mientras que una EEP 10 (esfuerzo máximo, extenuante), se ha demostrado que la EEP es un instrumento eficiente para niñoadolescentes, debido a que pueden ser capaces de regular la intensidad del ejercicio en medio de una sesión de acuerdo al 
esfuerzo percibido por cada uno (Yelling, Lamb, \& Swaine, 2002). Durante la aplicación del tratamiento, los sujetos debían realizar todos los ejercicios a una EEP entre 9 - 10. Una vez concluido el tiempo de trabajo (15 s), debían realizar una pausa activa caminando con una EEP entre 2 - 3. Posterior a esta pausa, comenzaban el siguiente ejercicio.

\section{ANÁlisis Estadístico}

Los datos fueron analizados de la siguiente manera y orden: las variables de estatura $(\mathrm{cm})$, peso $(\mathrm{kg}), \mathrm{IMC}$, altura de salto $(\mathrm{h})$ y los Picos de potencia $(\mathrm{Pp})$ de los miembros inferiores en el Test de Sargent, fueron sometidos a la prueba de normalidad Kolmogorov-Smirnov (K-S). Para comparar el comportamiento de las variables durante la aplicación del método de entrenamiento pliométrico basado en HIIT, se aplicó la prueba $t$ de Student. Con dicha prueba, se comparó el pre test y post test tanto para el GE como para el GC. Mientras que el tamaño del efecto (TE) se calculó utilizando la prueba d de Cohen. Este análisis considera un efecto insignificante $(\mathrm{d}<0,2)$, pequeño $(\mathrm{d}=0,2$ hasta 0,6$)$, moderado $(\mathrm{d}=0,6$ a 1,2$)$, grande $(\mathrm{d}=1,2$ a $2,0)$ o muy grande $(\mathrm{d}>2,0)$. El nivel de significancia para cada uno de los análisis estadísticos fue de $\mathrm{p}<$ 0,05. El análisis de los datos fue realizado a través de Microsoft Excel 2013.

\section{Resultados}

Una vez aplicada la prueba t de Sudent, el peso $(\mathrm{kg})$ y el IMC presentaron un aumento significativo entre el pre test y post test sólo para el GE ( $\mathrm{p}<0,001$; TE $=, 182$ y $\mathrm{p}<0,0001$; $\mathrm{TE}=, 287)$ respectivamente. Mientras tanto, en el GC, no se observaron cambios significativos en las mismas variables medidas $(\mathrm{p}=$ $0,34 ; \mathrm{TE}=, 009$ y $\mathrm{p}=0,34 ; \mathrm{TE}=, 010)$. Las progresiones y los cambios producidos entre el pre test y post test, se encuentran reportados en la Tabla 3.

TABLA 3

Características GE y GC en pre test y post test

\begin{tabular}{|c|c|c|c|c|}
\hline \multicolumn{5}{|c|}{ Grupo Control } \\
\hline & $\begin{array}{c}\text { pre test } \\
\text { media } \pm \text { DS }\end{array}$ & $\begin{array}{c}\text { post test } \\
\text { media } \pm \text { DS }\end{array}$ & t de Student & $\begin{array}{c}\mathrm{d} \text { de } \\
\text { Cohen }\end{array}$ \\
\hline Estatura $(\mathrm{cm})$ & $166,6 \pm 0,7$ & $166,6 \pm 0,7$ & ns & ,000 \\
\hline Peso (kg) & $64,3 \pm 9,7$ & $64,3 \pm 9,8$ & ns & ,009 \\
\hline IMC & $23,0 \pm 3,1$ & $23,0 \pm 3,1$ & ns & ,010 \\
\hline \multicolumn{5}{|c|}{ Grupo Experimental } \\
\hline & $\begin{array}{c}\text { pre test } \\
\text { media } \pm \text { DS }\end{array}$ & $\begin{array}{c}\text { post test } \\
\text { media } \pm \text { DS }\end{array}$ & $\begin{array}{c}\mathrm{t} \text { de } \\
\text { Student }\end{array}$ & d de Cohen \\
\hline Estatura $(\mathrm{cm})$ & $1,65 \pm 0,1$ & $1,65 \pm 0,1$ & ns & ,000 \\
\hline Peso (kg) & $61,1 \pm 13,5$ & $63,6 \pm 14,0$ & ** & , 182 \\
\hline IMC & $22,1 \pm 3,1$ & $23,1 \pm 3,2$ & $* * *$ & ,287 \\
\hline
\end{tabular}

GE (Grupo Experimental); GC (Grupo Control); cm (centímetros); kg (kilogramos); IMC (índice de masa corporal); DS (desviación estándar); ns (no significativo); ${ }^{* *} \mathrm{p}<0,001 ;{ }^{* * *} \mathrm{p}<0,0001$

En relación a la altura de salto $(\mathrm{h})$ en el Test de Sargent se presentó un aumento significativo entre el pre test y post test sólo para el GE ( $\mathrm{p}<, 0001 ; \mathrm{TE}=0,763)$. Mientras tanto, en el GC, no se observaron cambios significativos en la misma variable medida $(\mathrm{p}=0,08$; TE $=, 040)$. Por su parte, y en relación a los Pp, el GE presentó un aumento significativo entre el pre test y post test $(\mathrm{p}<0,0001 ; \mathrm{TE}=, 413)$. Mientras que el GC, no evidenció cambios significativos en el Pico de potencia $(\mathrm{Pp})$ calculado a través de la fórmula de Sayers $(\mathrm{p}$ $=0,13 ; \mathrm{TE}=, 052)$. Las progresiones y los cambios producidos entre el pre test y post test, se encuentran reportados en la Tabla 4. 
TABLA 4

Altura de salto y Pico de potencia obtenido a través del Test de Sargent y la fórmula de Sayers respectivamente para el GE y GC en pre test y post test

\begin{tabular}{|c|c|c|c|c|}
\hline \multicolumn{5}{|c|}{ Grupo Control } \\
\hline & $\begin{array}{c}\text { pre test } \\
\text { media } \pm \text { DS }\end{array}$ & $\begin{array}{c}\text { post test } \\
\text { media } \pm \text { DS }\end{array}$ & $\begin{array}{c}\mathrm{t} \text { de } \\
\text { Student }\end{array}$ & $\begin{array}{c}\mathrm{d} \text { de } \\
\text { Cohen }\end{array}$ \\
\hline $\mathrm{h}$ de salto $(\mathrm{cm})$ & $37,5 \pm 6,1$ & $37,8 \pm 6,3$ & ns & , 040 \\
\hline $\mathrm{Pp}(\mathrm{W})$ & $3.076,4 \pm 409,4$ & $3.098,3 \pm 430,5$ & ns & ,052 \\
\hline \multicolumn{5}{|c|}{ Grupo Experimental } \\
\hline & $\begin{array}{c}\text { pre test } \\
\text { media } \pm \text { DS }\end{array}$ & $\begin{array}{c}\text { post test } \\
\text { media } \pm \text { DS }\end{array}$ & $\begin{array}{c}\mathrm{t} \text { de } \\
\text { Student }\end{array}$ & $\begin{array}{l}\mathrm{d} \text { de } \\
\text { Cohen }\end{array}$ \\
\hline $\mathrm{h}$ de salto $(\mathrm{cm})$ & $38,9 \pm 4,9$ & $42,7 \pm 5,0$ & $* * *$ & 763 \\
\hline $\mathrm{Pp}(\mathrm{W})$ & $2.999,7 \pm 790,0$ & $3.329,1 \pm 806,0$ & $* * *$ & 413 \\
\hline
\end{tabular}

Test de Sargent; h (altura); cm (centímetros); Fórmula de Sayers; GE (Grupo Experimental); GC (Grupo Control); Pp (Pico de potencia); W (watts); DS (desviación estándar); ns (no significativo); ${ }^{* * *} \mathrm{p}<0,0001$.

\section{Discusión}

En relación con el objetivo principal del estudio, al término de la aplicación del entrenamiento pliométrico basado en un HIIT, el GE evidenció un aumento significativo en la altura (h) y Pp de salto. Sin embargo, un punto a considerar antes de la discusión, fue que ambos grupos recibieron durante toda la intervención un entrenamiento técnico-táctico orientado al fútbol escolar, y que solo el GE recibió adicionalmente al comienzo de las distintas sesiones, un protocolo pliométrico basado en HIIT. Por lo tanto, se podría considerar que la sumatoria de ambos trabajos (técnico-táctico + pliometría basada en HIIT), permitió aumentar la h y Pp de salto en el GE, no así el GC que solo efectuó el entrenamiento habitual técnico-táctico, no evidenciando cambios en la h y $\mathrm{Pp}$ de salto. Al igual que el presente estudio, existen otras investigaciones con protocolo en base a pruebas de campo que buscan el incremento en altura de salto y potencia muscular. Dentro de éstas, Chelly et al. (2010), luego de ocho semanas de entrenamiento pliométrico, evidenciaron un aumento significativo en la altura de salto en pruebas Squat Jump (SJ) - Counter Movement Jump (CMJ)(p < 0,01 y p < 0,001 respectivamente), mientras que en los Pp se reportaron incrementos significativos sólo en CMJ $(\mathrm{p}<0,05)$. Desafortunadamente, y pese a las significancias en las $\mathrm{h}$ de salto y los $\mathrm{Pp}$ reportadas, en dicha investigación no se utilizó el método pliométrico basado en HIIT. A su vez, Michailidis et al.(2013), posterior a 12 semanas de entrenamientos pliométricos, reportaron diferencias significativas en velocidad, salto, agilidad y fuerza de piernas $(\mathrm{p}<0,05)$. En otro estudio, se evidenció, luego de ocho semanas de entrenamiento pliométrico, un aumento significativo en el CMJ y en los Pp ( $<<0,05)$ (Ozbar et al., 2014). Si bien es cierto que los tres estudios mencionados anteriormente fueron realizados con futbolistas, existen otras investigaciones en donde se ha utilizado el método pliométrico para obtener aumento significativo en atletas velocistas, jugadoras de baloncesto, jugadores de balonmano, como también en mujeres gimnastas (Chelly et al., 2014; Hall, Bishop, \& Gee, 2016; Mackala, \& Fostiak, 2015; Roopchand-Martin, \& Lue-Chin, 2010). En cada una de estas investigaciones, en base al método pliométrico utilizado, se ha contribuido a mejorar la h de salto y los Pp en diferentes modalidades deportivas. Sin embargo, ninguna de estas investigaciones utilizó un entrenamiento pliométrico basado en HIIT para incrementar la potencia muscular, menos aún en futbolistas adolescentes.

Dada la evidencia anterior, se ha podido observar la importancia del desarrollo de la fuerza en las distintas modalidades deportivas (Huerta et al., 2016a; Huerta et al., 2016b; Chelly et al., 2010) y a su vez, la eficiencia de la pliometría para incrementar los niveles de potencia del tren inferior (Chelly et al., 2014). No obstante, también se requiere que los sujetos estén libre de lesiones. En un estudio desarrollado por Ter Stege, Dallinga, Benjaminse \& Lemmink (2014), indicaron que un entrenamiento neuromuscular a través de ejercicios pliométricos reduce el valgo de rodilla, mejora sus momentos de flexión y ángulos. A su vez, Xu, Lombardi, Jiao \& Banfi (2016), en una revisión sistemática, concluyeron que los ejercicios pliométricos aumentan el pico de masa ósea en las mujeres jóvenes, y que estos ejercicios también podrían 
ser útiles en los hombres, promocionando la salud ósea y la prevención de la osteoporosis. En otros estudios especializados en la aplicación de un HIIT con resultados de adaptación neuromusculares, se ha concluido que este tipo de entrenamientos aumenta el rendimiento de velocidad ( $\mathrm{p}<0,001$ ) (Kohn et al., 2011; Sperlich et al. 2011), fuerza explosiva en CMJ ( $\mathrm{p}<0,001)$ (Brocherie et al., 2015), lactato deshidrogenasa (LDH) homogeneizado $(\mathrm{p}<0,05)$ y fibras tipo IIa $(\mathrm{p}<0,05)$ (Kohn et al., 2011). Pese a los buenos resultados demostrados con el método HIIT en los deportistas (Kohn et al., 2011; Sperlich et al. 2011; Brocherie et al., 2015), ninguna de estas investigaciones mencionadas, incluyó ejercicios pliométricos dentro del HIIT, por lo que una adaptación neuromuscular producto de la combinación de estos métodos sigue quedando al debe. Por lo descrito en los párrafos anteriores, los beneficios del entrenamiento pliométrico y de un método HIIT han sido explícitos en el rendimiento deportivo, pero, al igual que el incremento de la h y los Pp a través de un entrenamiento pliométrico basado en un HIIT, los reportes de esta metodología innovadora de entrenamiento son escasos. En este sentido y hasta donde el conocimiento alcanza, sólo se ha investigado la combinación de este método en mujeres con obesidad y en corredores adultos recreativos (Ache-Dias, Dellagrana, Teixeira, Dal Pupo, \& Moro, 2016; Racil et al., 2016). Es así como Ache-Dias et al. (2016), reportaron incrementos significativos en la h de salto y los Pp en CMJ ( $\mathrm{p} \leq 0,05)$. Sin embargo, y a diferencia del presente estudio, estos investigadores no reportan progresiones en la intensidad ni en las cargas de trabajo. Por su parte, una población de mujeres adolescentes que padecían de obesidad, evidenció disminuciones significativas en aspectos bioquímicos, antropométricos, IMC, grasa corporal y circunferencia de cintura luego de haber realizado un entrenamiento pliométrico combinado con HIIT, sin embargo, presentaron un aumento significativo $(\mathrm{p}=0,021)$ de la masa corporal magra, a pesar de aquello, no miden la h de salto, como tampoco los Pp (Racil et al., 2016). Desafortunadamente, un entrenamiento pliométrico combinado con HIIT, no ha sido llevado a cabo en una población de futbolistas adolescentes.

Los resultados del presente estudio, dejan en evidencia que un entrenamiento pliométrico basado en un HIIT es una herramienta efectiva para incrementar la h de salto y los Pp en futbolistas adolescentes Sub- 17. Un uso correcto de este método de entrenamiento, permitiría obtener mayores niveles de rendimiento deportivo. Lo anterior, se debe principalmente a que un HIIT adicionado a ejercicios explosivos podrían mejorar la potencia y algunos factores relacionados con fatiga (Brocherie et al., 2015; Costigan et al., 2015).

\section{Aplicaciones Prácticas}

Los hallazgos de esta investigación, permitieron obtener beneficios en altura de salto y desarrollo neuromuscular a través de la potencia muscular de los futbolistas Sub-17 sometidos al plan de entrenamiento pliométrico basados en un HIIT. Es por ello, que se sugiere implementar este plan de entrenamiento con futbolistas adolescentes. De esta manera, cada uno de los individuos podrá vivenciar una mejoría en su altura de salto y potencia muscular, desempeñándose así de mejor forma tanto en los entrenamientos como en la competencia. Por otra parte, creemos que es factible desarrollar esta investigación en otras realidades, considerando otros segmentos de la población en estudio y a su vez con un tiempo de investigación más extenso, permitiendo realizar otras mediciones a los individuos, como componentes antropométricos y estimación del consumo máximo de oxígeno.

\section{LIMITACIONES}

Las limitaciones del presente estudio, han sido la ausencia de mediciones antropométricas. Cabe mencionar que el GE presentó un incremento del IMC, sin embargo, no podemos atribuir dicho acontecimiento a un aumento de masa grasa o masa magra. Aun así, la ganancia de aptitud muscular que han tenido los sujetos de nuestro estudio, puede estar asociada a una mejor salud independiente del IMC (Ingle, Stephenson, \& Sandercock, 2016). Por otra parte, los resultados de este estudio no necesariamente 
son extrapolables a otros contextos, pero si pueden dar indicios de un método efectivo y que debe seguir siendo desarrollado en futuras investigaciones.

\section{ReFERENCIAS}

Abe, T., Kitaoka, Y., Kikuchi, D. M., Takeda, K., Numata, O., \& Takemasa, T. (2015). High-intensity interval training-induced metabolic adaptation coupled with an increase in hif-1alpha and glycolytic protein expression. Journal of Applied Physiology, 119(11), 1297-1302. doi: https://10.1152/japplphysiol.00499.2015

Ache-Dias, J., Dellagrana, R. A., Teixeira, A. S., Dal Pupo, J., \& Moro, A. R. (2016). Effect of jumping interval training on neuromuscular and physiological parameters: A randomized controlled study. Applied Physiology, Nutrition, and Metabolism, 41(1), 20-25. doi: https://10.1139/apnm-2015-0368

Bautista, I.J., Chirosa, I.J., Chirosa, L. J., Martín, I., González, A., \& Robertson, R. J. (2014). Development and validity of a scale of perception of velocity in resistance exercise. Journal of Sports Science and Medicine, 13, 542-549.

Borg, G. (1998). Borg's perceived exertion and pain scales. Human kinetics.

Brocherie, F., Girard, O., Faiss, R., \& Millet, G. P. (2015). High-intensity intermittent training in hypoxia: A doubleblinded, placebo-controlled field study in youth football players. Journal of Strength and Conditioning Research, 29(1), 226-237. doi: https://10.1519/JSC.0000000000000590

Ceroni, D., Martin, X. E., Delhumeau, C., \& Farpour-Lambert, N. J. (2012). Bilateral and gender differences during single-legged vertical jump performance in healthy teenagers. Journal of Strength and Conditioning Research, 26(2), 452-457. doi: https://10.1519/JSC.0b013e31822600c9

Chelly, M. S., Ghenem, M. A., Abid, K., Hermassi, S., Tabka, Z., \& Shephard, R. J. (2010). Effects of in-season shortterm plyometric training program on leg power, jump- and sprint performance of soccer players. Journal of Strength and Conditioning Research, 24(10), 2670-2676. doi: https://10.1519/JSC.0b013e3181e2728f

Chelly, M. S., Hermassi, S., Aouadi, R., \& Shephard, R. J. (2014). Effects of 8-week in-season plyometric training on upper and lower limb performance of elite adolescent handball players. Journal of Strength and Conditioning Research, 28(5), 1401-1410. doi: https://10.1519/JSC.0000000000000279

Comfort, P., Stewart, A., Bloom, L., \& Clarkson, B. (2014). Relationships between strength, sprint, and jump performance in well-trained youth soccer players. Journal of Strength and Conditioning Research, 28(1), 173-177. doi: https://10.1519/JSC.0b013e318291b8c7

Costigan, S. A., Eather, N., Plotnikoff, R. C., Taaffe, D. R., \& Lubans, D. R. (2015). High-intensity interval training for improving health-related fitness in adolescents: A systematic review and meta-analysis. British Journal of Sports Medicine, 49(19), 1253-1261. doi: https://10.1136/bjsports-2014-094490

Davies, G., Riemann, B. L., \& Manske, R. (2015). Current concepts of plyometric exercise. International Journal of Sports Physical Therapy, 10(6), 760-786.

de Hoyo, M., Gonzalo-Skok, O., Sanudo, B., Carrascal, C., Plaza-Armas, J. R., Camacho-Candil, F., \& Otero-Esquina, C. (2016). Comparative effects of in-season full-back squat, resisted sprint training, and plyometric training on explosive performance in U-19 elite soccer players. Journal of Strength and Conditioning Research, 30(2), 368-377. doi: https://10.1519/JSC.0000000000001094

de Salles, P. G., Vasconcellos, F. V., de Salles, G. F., Fonseca, R. T., \& Dantas, E. H. (2012). Validity and reproducibility of the sargent jump test in the assessment of explosive strength in soccer players. Journal of Human Kinetics, 33, 115-121. doi: https://10.2478/v10078-012-0050-4

Ebben, W. P., Fauth, M. L., Garceau, L. R., \& Petushek, E. J. (2011). Kinetic quantification of plyometric exercise intensity. Journal of Strength and Conditioning Research, 25(12), 3288-3298. doi: https://10.1519/JSC.0b0 $13 \mathrm{e} 31821656 \mathrm{a} 3$

Franco-Marquez, F., Rodriguez-Rosell, D., Gonzalez-Suarez, J. M., Pareja-Blanco, F., Mora-Custodio, R., YanezGarcia, J. M., \& .Gonzalez-Badillo, J. J. (2015). Effects of combined resistance training and plyometrics on 
physical performance in young soccer players. International Journal of Sports Medicine, 36(11), 906-914. doi: https://10.1055/s-0035-1548890

Hall, E., Bishop, D. C., \& Gee, T. I. (2016). Effect of plyometric training on handspring vault performance and functional power in youth female gymnasts. PloS One, 11(2), e0148790. doi: https://10.1371/journal.pone.0 148790

Huerta, Á., Chirosa, L., Guisado, R., Chirosa, I., \& Cáceres, P. (2016a). Acute effect of a complex training protocol of back squats on 30-m sprint times of elite male military athletes. Journal of Physical Therapy Science, 28(3), 752. https://doi.org/10.1589/jpts.28.752

Huerta, A., Chirosa, L., Guisado, R., Chirosa, I., y Cáceres, P. (2016b). Efecto de la resistencia variable sobre la potenciación post activación: Una revisión sistemática. Arch Med Deporte, 33(5), 337-344.

Huerta, Á., Galdames, S., Cataldo, M., Barahona, G., Rozas, T., \& Cáceres, P. (2017). Efectos de un entrenamiento intervalado de alta intensidad en la capacidad aeróbica de adolescentes. Revista médica de Chile, 145(8), 972-979. https://dx.doi.org/10.4067/s0034-98872017000800972

Ingle, L., Stephenson, A., \& Sandercock, G. R. (2016). Physical activity profiles and selected muscular fitness variables in english schoolchildren: A north-south divide? European Journal of Sport Science, 16(8), 1187-1196. doi: h ttps://10.1080/17461391.2016.1183714

Kohn, T. A., Essen-Gustavsson, B., \& Myburgh, K. H. (2011). Specific muscle adaptations in type II fibers after highintensity interval training of well-trained runners. Scandinavian Journal of Medicine \& Science in Sports, 21(6), 765-772. doi: https://10.1111/j.1600-0838.2010.01136.x

Lara-Sanchez, A. J., Zagalaz, M. L., Berdejo-Del-Fresno, D., \& Martinez-Lopez, E. J. (2011). Jump peak power assessment through power prediction equations in different samples. Journal of Strength and Conditioning Research, 25(7), 1957-1962. doi: https://10.1519/JSC.0b013e3181e06ef8

Lloyd, R. S., Radnor, J. M., De Ste Croix, M. B., Cronin, J. B., \& Oliver, J. L. (2016). Changes in sprint and jump performances after traditional, plyometric, and combined resistance training in male youth pre- and post-peak height velocity. Journal of Strength and Conditioning Research, 30(5), 1239-1247. doi: https://10.1519/JSC .0000000000001216

Logan, G. R., Harris, N., Duncan, S., Plank, L. D., Merien, F., \& Schofield, G. (2016). Low-active male adolescents: A dose response to high-intensity interval training. Medicine and Science in Sports and Exercise, 48(3), 481-490. doi: https://10.1249/MSS.0000000000000799

Lorenz, D., \& Morrison, S. (2015). Current concepts in periodization of strength and conditioning for the sports physical therapist. International Journal of Sports Physical Therapy, 10(6), 734-747.

Loturco, I., Pereira, L. A., Kobal, R., Zanetti, V., Kitamura, K., Abad, C. C., \& Nakamura, F. Y. (2015). Transference effect of vertical and horizontal plyometrics on sprint performance of high-level U-20 soccer players. Journal of Sports Sciences, 33(20), 2182-2191. doi: https://10.1080/02640414.2015.1081394

Mackala, K., \& Fostiak, M. (2015). Acute effects of plyometric intervention-performance improvement and related changes in sprinting gait variability. Journal of Strength and Conditioning Research, 29(7), 1956-1965. doi: h ttps://10.1519/JSC.0000000000000853

McKay, D., \& Henschke, N. (2012). Plyometric training programmes improve motor performance in prepubertal children. British Journal of Sports Medicine, 46(10), 727-728. doi: https://10.1136/bjsports-2012-091417

Michailidis, Y., Fatouros, I. G., Primpa, E., Michailidis, C., Avloniti, A., Chatzinikolaou, A., \& Kambas, A. (2013). Plyometrics' trainability in preadolescent soccer athletes. Journal of Strength and Conditioning Research, 27(1), 38-49. doi: https://10.1519/JSC.0b013e3182541ec6

Naimo, M. A., de Souza, E. O., Wilson, J. M., Carpenter, A. L., Gilchrist, P., Lowery, R. P., \& Joy, J. (2015). Highintensity interval training has positive effects on performance in ice hockey players. International Journal of Sports Medicine, 36(1), 61-66. doi: https://10.1055/s-0034-1382054

Ozbar, N., Ates, S., \& Agopyan, A. (2014). The effect of 8-week plyometric training on leg power, jump and sprint performance in female soccer players. Journal of Strength and Conditioning Research, 28(10), 2888-2894. doi: https://10.1519/JSC.0000000000000541 
Racil, G., Zouhal, H., Elmontassar, W., Ben Abderrahmane, A., De Sousa, M. V., Chamari, K., \& Coquart, J. B. (2016). Plyometric exercise combined with high-intensity interval training improves metabolic abnormalities in young obese females more so than interval training alone. Applied Physiology, Nutrition, and Metabolism, 41(1), 103-109. doi: https://10.1139/apnm-2015-0384

Roopchand-Martin, S., \& Lue-Chin, P. (2010). Plyometric training improves power and agility in jamaica's national netball team. The West Indian Medical Journal, 59(2), 182-187. doi:1195

Sohnlein, Q., Muller, E., \& Stoggl, T. L. (2014). The effect of 16-week plyometric training on explosive actions in early to mid-puberty elite soccer players. Journal of Strength and Conditioning Research, 28(8), 2105-2114. doi: ht tps://10.1519/JSC.0000000000000387

Sperlich, B., De Marees, M., Koehler, K., Linville, J., Holmberg, H. C., \& Mester, J. (2011). Effects of 5 weeks of high-intensity interval training vs. volume training in 14-year-old soccer players. Journal of Strength and Conditioning Research, 25(5), 1271-1278. doi: https://10.1519/JSC.0b013e3181d67c38

Teo, S. Y., Newton, M. J., Newton, R. U., Dempsey, A. R., \& Fairchild, T. J. (2016). Comparing the effectiveness of a short-term vertical jump vs. weightlifting program on athletic power development. Journal of Strength and Conditioning Research, 30(10), 2741-2748. doi: https://10.1519/JSC.0000000000001379

Ter Stege, M. H., Dallinga, J. M., Benjaminse, A., \& Lemmink, K. A. (2014). Effect of interventions on potential, modifiable risk factors for knee injury in team ball sports: A systematic review. Sports Medicine, 44(10), 1403-1426. doi: https://10.1007/s40279-014-0216-4

Xu, J., Lombardi, G., Jiao, W., \& Banfi, G. (2016). Effects of exercise on bone status in female subjects, from young girls to postmenopausal women: An overview of systematic reviews and meta-analyses. Sports Medicine, 46(8), 1165-1182. doi: https://10.1007/s40279-016-0494-0

Yelling, M., Lamb, K. L., \& Swaine, I. L. (2002). Validity of a pictorial perceived exertion scale for effort estimation and effort production during stepping exercise in adolescent children. European Physical Education Review, 8(2), 157-175. https://doi.org/10.1177/1356336X020082007 